\title{
The Effectiveness of a Cognitive Behavioral Therapy Program in Reducing Tics (motor \& vocal) in a Sample of Children with Tourette Syndrome
}

\author{
Dr. Omar Khalil Mousa Atiyat, Dr. Abdulaziz Abdullah Alothman
}

Ph.D. In Special Education, Ministry of Education / Special Education Department, UAEDubai.Omar.Atiyat@moe.gov.ae

Associate Professor of Special Education, Majmaah University, School of Education, Department of Special Education. aa.alothman@mu.edu.sa

\begin{abstract}
The current study aims verifying a behavior modification program (BMP) effectiveness, based on cognitive behavior modification strategies, in reducing (motor and vocal) tic levels among individuals with Tourette syndrome .The study sample comprised (10) children with Tourette syndrome aging between (9-17 years old).To achieve the study goals, the experimental approach was followed. Research tool were developed by Yale Global Tic Severity Scale (YGTSS). The BMP consists of (12) sessions, one session per week and (60) minutes for each session. The study results indicated that there are significant statistical differences at ( $\alpha=0.05$ ) of performance level of the sample on the pre and post tests on the YGTSS motor, vocal, damage assessment, and on the overall totals in favor of the post test. No Statistically significant differences at $(\alpha=0.05)$ were found based on gender, age variables on tic levels (motor/vocal) of the sample in the post test. The results also indicated that there were no statistically significant differences $(\alpha=0.05)$ between performance means levels of the sample on the post and sequential tests which can be attributed to the behavior modification program effect on both (Motor/vocal) dimensions, on all sub-dimensions of each dimension including(number, frequency, intensity, complexity, overlap of tics), as well as damage assessment and the total score of the scale.
\end{abstract}

Keywords:

Tourette syndrome, Behavior Modification, Cognitive behavior Modification, Vocal tics, Motor tics

Article Received: 18 August 2020, Revised: 3 November 2020, Accepted: 24 November 2020

\section{The study problem and its questions:}

Syndromes often transform the mobility and vocalization shown by individuals with Tourette's syndrome between them and between achieving acceptable levels of self-satisfaction and social acceptance by others, which requires research into ways that would constitute a therapeutic approach to reduce the emergence of these Syndromes, whether by duration, Severity, or repetition. Through reviewing the theoretical literature and previous studies, the researcher noticed the lack of behavior modification and counseling programs that dealt with this category of individuals with neurobehavioral disorders in general and those related to reducing the level of appearance of motor and vocal Syndromes in those with Tourette's Syndrome in particular. And after the researcher had a previous research experience that was the first in the Arab world to target individuals with Syndrome Tourette - according to the limits of the researcher's knowledge and knowledge - through which he built a diagnostic scale for Tourette Syndrome and personally touched the suffering of these individuals, largely due to the lack of behavioral therapeutic programs that may be It was presented to them by specialists with the aim of reducing the effects of these behavioral symptoms on them, which may leave an impact on the acceptance and life of these individuals, and from here the idea of the current study emerged.

The current study came in an attempt to answer the following main question: What is the degree of effectiveness of a behavior modification program based on cognitive behavior modification strategies in reducing the level of Syndromes (motor and vocal) in a sample of individuals with 
Tourette's syndrome? By answering the following sub-questions specifically:

The first question: Are there statistically significant differences at the level of significance $(\alpha=0.05)$ between the average performance level of the study sample on the pre and post tests on the Yale global scale of Syndrome intensity attributable to the program?

The second question: Are there statistically significant differences at the level of significance $(\alpha=0.05)$ in the level of appearance of kinetic and vocal Syndromes among the study sample subjects in the post application due to the gender variable?

The third question: Are there statistically significant differences at the level of significance $(\alpha=0.05)$ in the level of emergence of kinetic and vocal Syndromes among the study sample subjects in the post application due to the variable of age?

The fourth question: Are there statistically significant differences at the level of significance $((\alpha=0.05)$ between the average performance level of the study sample on the post and tracer tests on the Yale Global Syndrome intensity scale attributable to the program?

\section{Study Justifications:}

Due to the increase in the number of individuals with Syndrome Tourette enrolled in various educational services and at various educational levels. After the increased levels of awareness and knowledge of Syndrome Tourette, it was imperative to research the provision of the best therapeutic educational programs based on behavioral modification procedures with various techniques and strategies that would help these students to face the problems they have related to the nature of their condition, whether on the sociopsychological or academic level. As a result of the increasing interest that Syndrome Tourette received and the lack of specialized studies and research that sought to research treatment strategies for people with Tourette's Syndrome, the researcher concluded the importance of addressing this topic to help workers in the field of providing treatment services to these individuals.

\section{Purpose of the study:}

The current study aims to verify the effectiveness of a behavior modification program based on cognitive behavior modification strategies in reducing the level of Syndromes (motor and vocal) in a sample of individuals with Tourette's Syndrome.

\section{The importance of study:}

The importance of the current study lies within both the theoretical dimension and the practical dimension, and the following is an explanation:

\section{Theoretical significance}

The theoretical importance of the current study lies in the scarcity of Arabic studies that have researched Syndrome Tourette, which contributes to supplementing the Arab library and the field of special education with a study that promotes research in the field of strategies for modifying the behavior of students with Tourette syndrome. It is also expected that the current study will pave the way for other scientific studies in the same field. With reference here to the fact that - according to the researcher's knowledge and the limits of his knowledge - there are only three Arab studies. The current researcher had one of them, which represented the first Arab study on Syndrome Tourette in (2015).

\section{Applied importance}

As for the practical importance of the current study, it can be highlighted by presenting a practical and effective behavior modification program that can be implemented in a relatively short time, and self-awareness. The results of the current study may also contribute to the process of directing the attention of educational experts, behavior modification specialists and psychological counselors to employ such strategies in providing services and specialized therapeutic educational programs based on various psychology theories.

Limits and limitations of the study:

- The current study was limited to (10) children who were diagnosed with a medical 
diagnosis, and a psycho-educational diagnosis that they had Tourette's Syndrome.

- The current study was limited to applying some cognitive behavioral strategies through the behavior modification program.

The results of this study are determined by the target study sample, the study methodology used and the tools used in it, namely: (Yale Global Tic Severity Scale (YGTSS), the cognitive behavioral modification program), and the extent of its validity and reliability.

\section{Terminology of study}

\section{- the program}

It is a set of organized and pre-planned procedures based on specific and clear goals that seek to modify behavior, and it is a tool prepared by the researcher and used in training Syndrome Tourette children in order to reduce their motor and vocal Syndromes. The program is defined procedurally in this study through the final image reached by the researcher Regarding the sincerity of the contents of the program, which was presented to a group of experts and specialists in the field of psychological counseling and special education, the program is implemented in (12) treatment sessions, each session is (60) minutes, at the rate of one session per week.

\section{- Behavior Modification:}

It is a process that includes shaping the behavior of the individual so that it seeks to reduce the rate of occurrence of unwanted behavior, or to eliminate and erase it on the one hand, and to support and reinforce the desired behavior on the other hand, by controlling the educational environment by applying the principles of learning in an organized manner. (Al-Khatib, 2019)

\section{Cognitive behavior modification:}

Spence and others (Spence, et, al, 2017) defined it as that type of behavioral therapy that focuses on exploring the relationships between a person's thoughts, feelings, and behavior. During cognitive behavioral therapy, the therapist actively works with the individual to uncover unhealthy thought patterns and how these thoughts may lead to the emergence of unwanted negative behaviors, through cognitive behavior modification techniques such as: self-monitoring, visualization, distraction, self-dialogue, stopping thoughts, Training in contrast to habit, training to solve problems, "defined procedurally as the ability of the behavior modification program that was developed and designed by the researcher to reduce the severity of motor and vocal Syndromes among the study sample individuals with Syndrome Tourette Spence, et al, 2017).

\section{Tourette's syndrome:}

The Diagnostic Statistical Manual of Mental Disorders, in its amended fifth edition, defined it as a neurological disorder that appears in childhood as the emergence of motor syndromes and / or vocal syndromes at different and complex levels. The effect of the drug on the physiology of a specific substance: cocaine or another medical condition, for example, Huntington's disease, and encephalitis. (APA, DSM5, 2013)

\section{Motor syndromes:}

They are frequent, involuntary movements that appear in short, rapid periods without prior warning. They are typical. These movements usually involve one group of muscles in the body. Like facial muscles, such as movements of the mouth and eyes, blinking of the eyes, staring or raising the eyebrows, or the neck, such as shaking the head forward and backward or twisting the neck or back, and shoulder to movements of the upper leaves or the lower extremities and the trunk. These movements may be complex or combine more than one involuntary movement pattern at the same time (Miltenberger, 2012).

Phonological syndromes

Attiyat (2015) defined them as involuntary movements that appear on short periods, typically once. It sounds like making unwitting noises such as hunching "loosening the throat," sniffing "taking out the nose" inappropriately, or tapping the tongue. And it appeared in some cases that he 
uttered the number of times the description of the image that appeared in the picture (Attiyat, 2015).

\section{Theoretical framework}

The process of developing and preparing treatment plans and programs for individual cases of Tourette's syndrome and implementing them in the early stages of life is an important factor in reducing the effects of the effects on these individuals and their families alike, and the procedures and treatment programs will schedule a set of methods, strategies and strategies for them. The emergence of the number of people suffering from this syndrome in school in regular schools or who receive educational services in educational centers, in a state of lack of awareness about Tourette syndrome, which leads to confusion between a number of other disabilities or other disabilities, and the lack of attention and activity Focus, specific, focus, focus on these cases. Psychological and educational therapeutic programs must be provided that have indications of honesty and constancy with the aim of working to place educational services in the same category. (Wood.\&, Piacentini, \& Walkup, 2010)

\section{Attiyat (2015) referred to Tourette} Syndrome (TS), also known as Tourette's disorder, as being a neurodevelopmental disorder characterized by the emergence of multiple motor tics and vocal syndromes in a chronic and persistent manner for a period of no less than 12 months. Its symptoms usually begin in early childhood, and reach It reaches its climax in preadulthood, and weakens later in adolescence. The characteristics of individuals with Syndrome Tourette vary, as well as their abilities and skills. Although there are common basic characteristics between them, the symptoms and characteristics that refer to them appear in the form of many and overlapping patterns that vary from simple to severe. Many different names have been given to these symptoms, such as: Syndrome, tics, and tics, Syndromes.

Tourette's Syndrome is among the most complex neurodevelopmental disorders in the group of tics disorders. Also, mental disorders such as obsessive-compulsive disorder (OCD) and attention deficit hyperactivity disorder (ADHD) often appear in many cases of Syndrome Tourette (Zeitlin, et al, 2001)

Recent studies show the syndrome resulting from TSN in up to $1 \%$ of children (Robertson et al 2009). (American Psychiatric Association (APA), 2013),However, while tics are multifunctional in cases of vocal tics. Tic disorder

The index has mentioned the statistics of mathematics and mentality in its amended fifth evidence

1. The emergence of more than one motor syndromes, with one or more vocal syndromes, they did not appear at the same time.

2. The emergence of a problem that may have arisen from the beginning.

3. These symptoms appear before the age of 18 years.

4. This medicine is not attributed to the physiological effects of a specific substance such as: (cocaine) or another medical condition such as infection (with Huntington's disease, encephalitis), for example.

(American Psychiatric Association, DSM5, 2013)

Recent studies related to the incidence of TSDs have shown that they are far from rare, as TS may appear in up to $1 \%$ of children (Robertson et al. 2009). Involuntary Syndromes are referred to as sudden, non-rhythmic vocal movements or sounds that are relatively common among individuals with Tourette's Syndrome of school age (American Psychiatric Association (APA), 2013) However, when single or multiple Syndromes persist for longer than a year, consideration should be given to diagnosing a disorder Tic. Chronic or persistent tic disorder is characterized by the presence of either one or multiple movements or vocal symptoms, but not both, that has lasted longer than 12 months (McGuire, 2016)

The Diagnostic and Statistical Manual of Mental Disorders, in its amended fifth edition, lists four basic criteria for the diagnosis of 
Tourette Syndrome, and these criteria are as follows:

1. The appearance of two or more motor Syndromes, with one or more vocal Syndromes, although not necessarily at the same time.

2. The emergence of some tics, which have lasted for more than a year since the onset of Syndromes.

3. These symptoms appear before the age of 18 years.

4. This disorder is not attributed to the physiological effects of a specific substance, such as: (cocaine) or any other medical condition such as infection (Huntington's disease, encephalitis), for example.

(American Psychiatric Association, DSM5, 2013)

These symptoms often appear during childhood after the age of six years, and reach their maximum in adolescence with an increase in the number, type, and frequency of these Syndromes (Bloch \&Leckman, 2009).

The symptoms and signs that can be considered as a primary indicator of the infection of Tourette's Syndrome most of the time are facial motor Syndromes, that is, that appear in the face such as rapid blinking in one or both eyes, spasms of the mouth and lips, wrinkling or tightening of the nose, frowning of the forehead and eyebrows, shaking of the head, in addition to The emergence of some vocal Syndromes and are intended for those involuntary sounds, such as hissing, "clearing the throat," or inhaling "exhaling" from the nose inappropriately, clicking on the tongue. In some cases, complex "verbal" vocalisations may appear, such as the echo "by repeating the words or phrases that he utters." Also, some cases may show a pattern of complex motor Syndromes such as: jumping, sitting in a squatting position, rotating around the soul. In some cases, self-harm behavior may appear, such as self-biting, head banging, and other types of self-harm behavior. Lebowitz, et al, 2012)
The majority of individuals with Tourette's Syndrome show comorbidities, the most common of which are obsessive-compulsive disorder (OCD), attention deficit hyperactivity disorder (ADHD (Cavanna et al, 2009)), and other behavioral problems and disorders such as emotional and sleep disorders. Less than one-third of individuals with Tourette Syndrome have selfharm behaviors of varying patterns and levels such as head banging and / or hitting or scratching.Self-harmful behaviors may be an integral part of Tourette syndrome as they are sometimes present even in simple cases, which necessitates controlling these symptoms to maintain In addition, a large proportion (possibly up to $30 \%$ ) of those with Tourette syndrome exhibit some symptoms of socially inappropriate behavior (NOSIS). These socially inappropriate symptoms include uttering profanity and shamelessness that results in insulting others or behaving. Aggressively, this may sometimes lead to a physical confrontation and confrontation with the law (Clare, et al, 2011).

And based on the behavioral symptoms mentioned by those individuals who have been diagnosed as suffering from Tourette's Syndrome, these individuals often face many difficulties in different areas of life, which increases the burdens placed on the shoulders of parents, family and caregivers, These individuals report a number of difficulties in the home, school or work environment alike. The individual may have some involuntary motor and vocal Syndromes inside the home, which may irritate the family and cause them a degree of inconvenience or embarrassment from visitors or neighbors in many cases (Steketee, 2011).

Within the school environment, these vocal, motor, and involuntary spastic movements create an atmosphere of chaos in the classroom, which may be a source of inconvenience to both colleagues and teachers alike, and these individuals may continue to suffer in the future, right up to the work environment, if the employers understand the nature of Their Status (Robertson, 2015) 
Hence the need for effective treatment interventions for individuals suffering from Syndromes in general and Tourette Syndrome in particular in order to be able to control these symptoms and it is possible to point out here that the treatment of Tourette Syndrome is a complex and intertwining matter because of the set of symptoms associated with this disorder and the diversity in the nature of the individual manifestations and symptoms of each status. With regard to the effectiveness of the medications being used to relieve the symptoms of Tourette Syndrome, studies clearly indicate that no drug agent has yet been identified that works to reduce tics in all patients. Tudor, et al, 2018)

While there are some calls for a trial and error method to determine the most appropriate treatment group for those suffering from the more complex group of symptoms from TS, such as those that show features of OCD or ADHD, this precludes the application of those aspects related to interactions, Pharmacokinetics and contraindications, plus concerns regarding the use of the drug in younger children. Clare, et al, 2011)

With reference to the possibility of using a number of non-drug therapies to treat moderateintensity Tourette syndrome. Here he intends the behavioral methods that may help in treating involuntary Syndromes, as many of these methods have succeeded in inhibiting involuntary Syndromes, both in their motor and vocal parts, for long periods of time (Bryson, et al, 2010), which would require the provision of the necessary therapeutic interventions to reduce the negative effects of These behavioral symptoms accompany this disorder, and psychotropic medications such as antipsychotics, alpha stimulants, anti-convulsions and convulsions have long been used in the treatment of tics (Huys, et al, 2012))

Although these antipsychotics are often effective in reducing the appearance of these symptoms, they often fail to eliminate them completely, with their association often with a range of negative side effects on their users. Which called for the recognition of the desired benefit of behavioral interventions and the tendency to adopt them as therapeutic strategies concurrent with medical-pharmacological therapeutic intervention, and then to be satisfied with behavioral therapy independently and from these therapeutic behavioral strategies, training strategies against habit (HRT). It is indicated here that training against habit is It is a short-term behavioral intervention; through self-awareness training, self-monitoring, and competing response training, it can also be easily adapted to include relaxation training using progressive muscle relaxation and breathing exercises to help reduce levels of anxiety and stress associated with an individual's attempt to control involuntary Syndromes (Franklin, et al, 2011)

The use of behavioral therapy programs to manage and reduce the incidence of non-adaptive behaviors in individuals with autism spectrum disorders and / or those with Tourette's syndrome and many other disorders is referred to here. These interventions included employing reinforcement strategies such as differential reinforcement of other behaviors (DRO) and differential reinforcement of alternative behavior (DRA), as well as the use of exclusion, selfawareness development, self-monitoring, and over-correction. Earles, \&, Myles, 1994)

This behavioral intervention strategy used with individuals with Tourette Syndrome has been called a "Comprehensive Involuntary Conflict Behavior Intervention Strategy (CBIT)" which includes teaching these individuals, their families and their teachers what makes motor or vocal Syndromes less or more severe, and how to organize the surrounding environment to help the individual. On the management of involuntary Syndromes, in addition to how the individual interacts with the stimuli that precede tics in the environment or even the stimuli or signals that come from within the individual's body, CBIT intervention is usually trained for the individual and the family through a psychologist, nurse, social worker, or other Therapists and service providers (Woods et al., 2010) 
Cognitive - Behavior Therapy (CBT) is characterized by that it deals with different disorders from a tripartite perspective as it deals with each of the cognitive, emotional and behavioral components of the disorder, which contributes to the diversity of therapeutic techniques used within this therapeutic model (cognitive, emotional, and behavioral techniques) in addition to its use (Conte, 2009).

Cognitive processes have a fundamental role in modifying cognitive behavior, even if they are not directly observable, as the concept of cognitive behavior modification refers to those mechanisms that are specifically designed to provide these individuals with the necessary means to self-control their behavior such as (cognitive modeling, self-reinforcement, selfmonitoring, and evaluation). Self-help, problemsolving skills, self-instruction (Miranda, \& Soriano, 2002)) with the possibility of modifying the apparent behavior of these individuals by teaching them to think in an appropriate manner and to observe their own behavior and the possibility of providing them with self-control skills and the ability to change and modify their behavior more effectively (Al-Khatib, 2003)

Cognitive behavioral therapy aims to achieve a set of goals in dealing with the situation, and among these goals is to work to reduce the feelings of distress and helplessness that the individual feels because of what he suffers from disorder, as well as aims to reduce the severity of emotional disorder and support the individual's active participation in social situations And to work to prevent relapses after completing the treatment program. It achieves this by following cognitive, behavioral and emotional strategies. The number of treatment sessions and the duration of the program depend on the severity and complexity of the condition or disorder and the individual's desire to establish a cooperative therapeutic relationship with the therapist (MacManus, \& Wait, 2009).

Based on this, the researcher designed a treatment program for individuals with Tourette's Syndrome, which aims to reduce the emergence of motor and vocal Syndromes and reduce their level of severity through cognitive behavioral therapy strategies, in addition to providing them with coping skills strategies that aim to improve their quality of life and improve the general performance of people with Syndrome Tourette.

\section{Second: Previous studies}

The researcher referred to a number of previous studies related to the study variables, whether those that looked at modifying the behavior of people with Tourette Syndrome through cognitive behavior modification strategies, or those that researched cognitive behavior modification strategies in controlling the behavior of individuals with autism spectrum disorder and other groups Special education, whose condition is often accompanied by some manifestations of adaptive behavior. This provided the researcher with a solid theoretical base from which he proceeded to examine the problem of the current study.

As for the study conducted by Tudor et al., 20018), it aimed, by following the one-case study approach, to know the effect of a cognitive behavior modification program to reduce anger and aggression behavior in a 9-year-old girl with Tourette Syndrome. She has some symptoms of general anxiety and the current study tool is represented by a cognitive behavioral modification program consisting of (12) sessions of cognitive behavioral therapy directed at managing anger and aggression, with selected modifications related to the context of controlling involuntary movements, over a period of (12) weeks that each child underwent And her mother. The results of the post evaluation indicated a significant and significant decrease in the child's anger attacks and aggressive behavior, in addition to a decrease in her convulsions and involuntary movements. The results of the study also indicated the continuing effect of the behavioral treatment program during the follow-up phase.

McGuire (2016) conducted a study that aimed to measure the effect of a behavior modification program to reduce the severity of motor and vocal Syndromes among members of 
Tourette syndrome. The study followed the onecase study approach, and the study sample was represented by a 14-year-old girl suffering from motor and vocal Syndromes at an average level According to the Yale Global Syndromes Severity Scale (YGTSS), the study tools were represented by the Yale Global Syndromes Severity Scale (YGTSS), and the behavior modification program designed for the purposes of reducing motor and vocal Syndromes using the habit reversal strategy distributed over (20) treatment sessions over a period of (20) weeks. At the rate of one session per week, the results of this study indicated that there are significant differences for a decrease in the incidence of motor and vocal Syndromes and a decrease in the social, physical and academic damages resulting from them.

In a study conducted by Assaf and others (2013), which aimed to identify the effect of a counseling program based on cognitive behavioral modification in reducing the aggressive behavior of a sample of students. The researchers followed the descriptive field and analytical approach to collect data on the study population to know the effect of applying the collective counseling program on modification. Cognitive behavior in reducing the aggressive behavior of the members of the sample, which consisted of (51) students and its students from the third and fourth grades of basic, from one of the Palestinian schools, and the study tools were represented by the aggressive behavior monitoring list and the behavior modification program, while the study procedures consisted of applying a list Monitoring the aggressive behavior of the members of the study population for the purposes of identifying the sample members who obtain a score of (60) or above and then subject these individuals to the cognitive behavioral program, and the results of the study indicated the presence of significant differences on the results of the members of the experimental group before and after the treatment procedures in favor of the treatment program The results also indicated that there were no statistically significant differences in terms of the effect of applying the program on modifying cognitive behavior in reducing the aggressive behavior of students. Attributed to the variable of sex.

While the aim of the study by each of McGinty and others (McGinty, et al, 2012) to determine the effect of modification of cognitive behavior in reducing the intensity of involuntary movements in a young man with Syndrome Tourette at the age of (22) years. The researchers used the one-case study approach. The study tools were represented by the SyndromeYale Global Tic Severity Scale (YGTSS) and the behavior modification program consisting of (10) sessions at one session per week during which the cognitive behavior modification procedures represented by habit reversal strategy, cognitive restructuring, and problem solving were used. The study procedures consisted of applying the Yale Global Syndrome Severity Scale (YGTSS), monitoring the severity of Syndromes in the case, determining the baseline, and then initiating the implementation of the behavior modification program procedures. The results of the study indicated that the case showed a significant improvement in reducing the level of involuntary movements as evidenced by a decrease His score on the Yale Global Tic Syndromes Severity Scale score from (16) at baseline to (8) after posttreatment and undergoing the behavioral program. The study also indicated that the effect of treatment will continue throughout the follow-up phase, which lasted for 4 months.

Al-Batayneh and Arnous (2011) also carried out a study aimed at identifying the effect of a proposed behavior modification program in reducing behavioral patterns among a sample of autistic children. The study sample consisted of three children with autism spectrum disorders enrolled in a special education center in the residential system, their ages ranged from Between (5-10) years, the study tools were represented by the behavioral observation form prepared by the researchers, which included (33) behaviors among autistic children, in addition to a behavior modification program aimed at reducing the behavioral patterns of autistic children. The 
researchers used the following behavior modification procedures: differential reinforcement of alternative behavior, exclusion, and sensory alienation. The program consisted of (90) sessions applied by the teacher in the playroom, where each session applied in the lesson room was followed by a session applied by the teacher in the playroom. The duration of the behavior modification session ranged between (25-35) minutes per session, at the rate of (3) sessions per day. The results of the study indicated a decrease in the frequency of all behavioral patterns found in each of the three children, which indicates the effectiveness of the behavioral procedures that were followed in the program with the aim of reducing the targeted behavioral patterns among the study sample.

As for Bryson and others (Bryson et, al, 2010), they conducted a study aimed at determining the effect of an integrated behavioral therapy program in treating Tourette Syndrome at the complex level and its accompanying symptoms. The study followed the one-case study approach, and the study sample was represented by a (49) year old woman, She suffers from motor and vocal Syndromes, and the study tools were represented by the Yale Global Tic Severity Scale and the behavior modification program consisting of (4) sessions with one session every two weeks during which behavior modification procedures represented by the strategy of reversing habit and relaxation were used, and the study procedures consisted of applying the Yale scale Global Syndrome Severity (YGTSS) on the condition, monitoring the severity of its Syndromes, determining the baseline, and then starting the implementation of the behavior modification program procedures. The study also indicated that the effect of treatment will continue throughout the follow-up phase, which lasted for 3 months.

Through the review of previous studies, it becomes clear to us that a group of studies have been presented that investigated modifying the behavior of individuals with Tourette Syndrome, and others aimed at modifying the behavior of individuals from other groups, which were presented as studies that sought to modify the behavior of the target groups in them through the application of modification procedures Different cognitive behavior. These studies varied in terms of targeted behavior and samples, the methodology used, in addition to the study tools and strategies used within the behavior modification programs. In terms of behavior and target groups, the studies conducted by Sobhi (2018), McGinty et al. (McGinty, et, al, 2012) aimed to reduce motility Syndromes in a sample of those with Tourette's Syndrome. As for the studies of McGuire (2016) and Bryson et al. (Bryson et, al, 2010), they aimed to reduce the severity and rate of occurrence of motor and vocal Syndromes in individuals with Tourette's Syndrome. As for the study (Tudor, et, al, 2018), it sought to reduce anger behavior and aggressive behavior in people with Tourette's Syndrome. While the study of Assaf and others (2013) aimed to modify the behavior of a sample of ordinary students who showed aggressive behavior, as for the study of Batayneh and Arnous (2011), it targeted modifying the behavior of (3) children with autism. The current study was different from the previous studies that were presented in that it targeted both kinetic and vocal Syndromes for individuals with Tourette Syndrome of both sexes within the age group (9-17) years.

As for the tools used in the study, the current study agreed with all the previous studies that were presented that targeted Syndrome Tourette individuals, as the study tools included the Yale Global Tic Severity Scale, and the behavior modification program, with different behavior modification techniques used by the program To another, as previous studies relied on the techniques of habit reversal and relaxation, while the current study used the techniques of cognitive behavior modification represented by the strategy of self-monitoring and self-control, the strategy of self-instruction and positive selftalk, immunization against stress, problem solving, and cognitive reconstruction, Distraction strategy (reciprocating palmistry), using the stimulus control strategy. 
In terms of the research methodology followed, the researcher followed in the current study the experimental method, and in that he agreed with the studies of Subhi (2018), Batayneh and Arnous (2011), while each of McGinty and others followed (McGinty, et, al, 2012), McGuire (2016), Bryson et al (2010) and Tudor et al. (Tudor, et, al, 20018) The one-case study approach. As for the study of Assaf and others (2013), it followed the descriptive approach.

\section{Search procedures}

\section{Study methodol}

The researcher followed the experimental approach "Experimental Design", which is based on the study of the relationship between the independent variable, which is the behavior modification program, and the dependent variable variable, which is represented by the kinetic and vocal syndromes in children with Tourette syndrome,before and after, as the researcher seeks to verify the effectiveness of the behavior modification program in reducing motor and vocal Syndromes among the study sample individuals with Tourette syndrome.

\section{Second: the study population and sample}

1. Study population: The case study population consisted of the total number of students diagnosed with Tourette syndrome and those receiving behavioral therapy services in (8) centers specialized in providing educational and psychological services. The total number of Syndrome Tourette cases that reviewed these eight centers periodically reached (28) cases from Different age groups.

2. The study sample: The study sample consisted of (10) individuals with Tourette's syndrome. They were chosen randomly, as the study sample consisted of (6) males and (4) females, whose ages ranged between (9-17) years, and Table No. (1) Represents the distribution of the study sample according to the variables of age group and gender.

Table (1) the individuals who represented the study sample and their distribution according to the study variables

\begin{tabular}{|c|c|c|c|}
\hline \multicolumn{2}{|c|}{ Number } & \multicolumn{2}{c|}{ Age } \\
\cline { 1 - 2 } Females & Males & $9-11$ & 1 \\
\hline 1 & 2 & $12-14$ & 2 \\
\hline 2 & 2 & $15-17$ & 3 \\
\hline 1 & 2 & & Total \\
\hline 4 & 6 & & \\
\hline \multicolumn{2}{|c|}{10} &
\end{tabular}

\section{Study instruments:}

The researcher used the following tools for the purposes of the study:

\section{Yale Global Tic Severity Scale (YGTSS)}

The researcher has used this scale in order to determine the motor and vocal Syndromes that appear in the study sample, the history of their occurrence, and their level of severity, as the YGTSS scale is a standard tool aimed at identifying symptoms of attention and impulsivity disorders such as Syndromes Disorder (TD) and Syndrome Tourette (TS) Obsessive-compulsive disorder (OCD) for children between (6-17) years old. (Sukhodolsky, et al, 2017)

The (YGTSS) scale is one of the most common scales for measuring the severity of Syndromes and the resulting damage. The scale is applied initially by making observations about the presence of motor and vocal Syndromes based on information from the respondent and behavioral observation. The examiner then evaluates the severity of "kinetic and vocal" Syndromes in five separate dimensions (number, frequency, intensity, complexity, and overlap). The scale is applied during two separate sessions, in the first session the case data is written and the case is observed during the session, and this session takes between (15-20) minutes and in the second session the scores are calculated and collected on the scale dimensions and this session usually takes between (10-15) (Kircanski, et al, 2010)

2. Cognitive behavioral modification program 
In order to achieve the objectives of the study, the researcher prepared a behavior modification program aimed at reducing motor or vocal Syndromes in a sample of children with Tourette's Syndrome according to a format consistent with the nature of the procedures and strategies derived from the behavior modification procedures. And represented in the strategy of self-monitoring and self-control in its three stages to deal with the disorder (self-monitoring, behavioral contracting with the self, reinforcement or punishment of the self), muscle and mental relaxation, in addition to the use of the selfinstruction strategy and positive self-talk as a strategy for dealing with supplies, and immunization against stress And cognitive restructuring, using the distraction strategy (reciprocal cessation) to deal with different supplies, using the strategy of controlling the stimulus, and it is intended to rearrange (organizing) the environment on the part of the individual in order to reduce some of his behaviors, and this program may consist of (12) treatment sessions In series, the duration of each session is (60) minutes, at the rate of one session per week.

Validity and reliability of the study tools

The first study tool was represented by the Yale Global Tic Severity Scale (YGTSS) in its Arabic version, which was prepared by Zoromba\& El-Gilany, 2018 (), and the necessary indications of validity and reliability were provided, while the second study tool was the behavior modification program The program was presented, in its initial form, to five faculty members, professors of special education and counseling at (Zayed University, the University of Jordan, Al-Balqa Applied University) in addition to six experts and specialists working in the field for the purposes of judging the program and judging its suitability in treating kinesthetic and vocal Syndromes. Individuals with Syndrome Tourette and in light of this, amendments to the program were implemented according to the opinions of the arbitrators.
Initially, the study sample individuals were randomly selected from a group of cases diagnosed with Syndrome Tourette and those wishing to receive behavioral therapeutic services from a specialized center, and their data collected, and then the researcher applied the Yale Global Tic Severity Scale (YGTSS) and monitored the degrees of the sample members On the scale (premeasurement) to represent the baseline, and then start the treatment program sessions consisting of (12) separate treatment sessions, at the rate of one session per week with a duration not exceeding (60) minutes per session.

After completing the implementation of the treatment program, the researcher intended to conduct a (telemetry) by re-applying the Yale Global Tic Severity Scale (YGTSS) to the study sample individuals after receiving the treatment program (the behavior modification program) and then work on analyzing the data statistically, and then A follow-up process took place for the cases for a period of (8) weeks, and conclusions were drawn in order to be presented, discussed and made appropriate recommendations in light of the study mechanism.

\section{The results:}

This chapter includes a comprehensive presentation of the research results after applying the study tools to the study sample, and then discussing them, in addition to presenting a number of conclusions, recommendations, and proposals

This study aimed to investigate the effectiveness of a treatment program based on cognitive behavior modification strategies in reducing both "motor and vocal" Syndromes in a sample of children with Tourette's syndrome, by comparing the pre-performance of the sample individuals on the Yale Global Syndromes Severity Scale (YGTSS), with their level of performance,dimensional after undergoing a behavior modification program based on cognitive behavioral procedures, and tracking its impact. 
The following is a presentation and discussion of the results related to the current study questions.

The first question: Are there statistically significant differences at the level of significance $(\alpha=0.05)$ between the performance level of the study sample on the pre and post tests on the dimensions of the Yale Global Syndrome intensity scale attributable to the program?

To answer this question, the Wilcoxon Signed Ranks Test was used to find the significance of the differences between the average ranks of the performance level of the study sample on the pre and post tests on the dimensions of the Yale Global Syndromes Severity Scale.

The results of the Wilcoxon Signed Ranks Test for the level of performance of the study sample on the pre and post tests on the dimensions of the Yale Syndromes Severity Scale

There were statistically significant differences at the level of significance $(\alpha=0.05)$ for the level of performance of the study sample on the pre and post tests on the Yale Global Scale for Syndromes (Kinetic Syndromes) severity, and the differences came in favor of the post application in all dimensions of the scale except for the number dimension that came with a significance level (0.083 ). As for acoustic Syndromes, it is evident from Table (2) that there are also statistically significant differences at the level of significance $(\alpha=0.05)$ for the level of performance of the study sample on the pre and post tests on the Yale Global Scale of Syndromes Severity (Acoustic Syndromes). The differences came in favor of the application,the dimension in all dimensions of the scale except for the number dimension that came with a significance level (0.317). As for each dimension of assessing the damage, it came with a significance level (0.004), while the final total on the scale came with a significant level (0.005), which indicates the existence of differences At the level of significance $(\alpha=0.05)$, it is attributed to the benefit of the program in the post application.

The results indicated that there were statistically significant differences at the level of significance $(\alpha=0.05)$ on the motor dimensions and acoustic Syndromes on all sub-dimensions of each dimension including (number of Syndromes, frequency of Syndromes, severity of Syndromes, complexity of Syndromes, overlap of Syndromes), as well as after evaluation. The harm, and the total score on the scale, is attributable to the effect of the behavior modification program. The results of the current study are consistent with what has been mentioned by some previous references and studies about the effectiveness of cognitive behavioral strategies in reducing both motor and vocal Syndromes in individuals with Tourette's Syndrome. The studies mentioned the importance of using the procedures and strategies adopted by the researcher, which are derived from cognitive behavior modification procedures. The strategy of self-monitoring and self-control in its three stages (self-monitoring, behavioral contracting with the self, strengthening or self-punishment), to deal with various behavioral disorders, especially those associated with Syndromes and Syndrome Tourette disorders, because of their effective role in organizing the individual's cognitive structures, and organizing his thoughts, which would lead That reflects positively on the individual's ability to deal with Syndromes, whether they are motor or vocal, by making him more aware of the nature of these Syndromes, and the mechanisms of overcoming, controlling and reducing them to the maximum extent possible.

In addition, following various strategies such as the self-instruction strategy and positive self-talk as strategies for dealing with Syndromes, immunization against stress, and cognitive reconstruction, through which the environment was rearranged on the part of the individual in order to reduce the level of his unacceptable behaviors, was a procedure. It is curatively effective in a number of previous studies, as it is also the case in the current study. The results of the current study were consistent with those of the findings of the study by Tudor et al. (20018), the study (Sobhi et al., 2018), the study of McGuire, 2016), and the study of McGinty et al. (al, 2012), and the study of Bryson et al. (Bryson et, al, 
2010). Looking also at those studies that were mentioned in the previous studies for having a common approach to adopting cognitive behavior modification strategies, which is represented in the study of (Assaf et al., 2013) ), Which aimed to reduce the aggressive behavior of a sample of normal children, and the study (Al-Batayneh and Arnous, 2011), which aimed to reduce the nonadaptive behavior patterns of a sample of children with autism disorder, we see that it also came in line with the effectiveness of cognitive behavioral measures in Reducing the unwanted behavioral patterns of the ordinary and people with special needs alike.

As for the second question, which reads: Are there statistically significant differences at the level of significance $(\alpha=0.05)$ in the level of kinetic and vocal Syndromes among the subjects of the study sample in the post application due to the gender variable? The researcher used to answer this question the Mann-Whitney test. To find the significance of the differences in the level of motor and vocal Syndromes among the study sample subjects in the post application depending on the gender variable.

Results of the Mann-Whitney test to find the significance of the differences in the level of motor and vocal Syndromes among the study sample subjects in the post application according to the gender variable

It is evident from the analysis that there are no statistically significant differences at the level of significance $(\alpha=0.05)$ due to the sex variable in the level of "kinetic Syndromes" which came with the level of significance (0.914) and vocal Syndromes that came with the level of significance (0.53), among the study sample members in the post application. The researcher explains that there are no significant differences due to the gender variable to what has been indicated by many studies that have investigated treatment programs for individuals with Tourette Syndrome, to the extent that the effectiveness of treatment programs provided to these individuals is not related to the sex of the individual or child as much as it is related to the nature and level of the individual's individual abilities. Whether it is male or female, and the nature of the content of the treatment program and the mechanisms of implementing the treatment program. This researcher intended to include the gender variable among the study variables, given that none of the previous studies addressed the effect of the gender variable on the effectiveness of behavior modification programs for individuals with Syndrome Tourette. , Noting that the only previous study that included males and females in its sample is the study (Subhi, 2018), as its study sample consisted of (3) females out of (13 participants) who had Syndrome Tourette and did not It deals, in turn, on the effect of the gender variable, which would be calculated in favor of the current study through the addition that it presented in the field of research. On the other hand, the researcher wanted to see the accuracy of the prevailing belief among some that females with Tourette's Syndrome respond better to treatment programs based on the fact that the prevalence of Tourette Syndrome among females is lower than that of males by an estimated rate of (3-4/1) for males compared to females. The results of the study dispelled this belief that there are no significant differences attributed to the gender variable on the program.

As for the third question, which reads: Are there statistically significant differences at the level of significance $(\alpha=0.05)$ in the level of kinetic and vocal Syndromes among the study sample members in the telemetry due to the age group variable?

To answer this question, the researcher resorted to using the Kruskal-Wallis Test to find the significance of differences in the level of motor and vocal Syndromes among the study sample members in the post application according to the age group variable.

The results of the Kruskal-Wallis Test to find the significance of the differences in the level of motor and vocal Syndromes among the study sample subjects in the post application according to the age group variable. 
It is evident that there are no statistically significant differences at the level of significance $(\alpha=0.05)$ due to the age group variable in both the motor Syndromes level, which came with a significance level (0.248) and the acoustic Syndromes that came with a significant level (0.435) among the study sample members in the post-measurement. The researcher believes that these results were logical due to the researcher's following the appropriate treatment procedures that are appropriate within the requirements of their implementation for the target age group between (9-17) years, due to the specificity of cognitive behavioral strategies such as the strategy of self-monitoring and self-control in its three stages (self-monitoring, Behavioral contracting with the self (self-reinforcement or punishment) and the possibility of applying and adapting it easily and easily without any complication that would negatively affect the performance of the individual in light of his chronological age on the behavior modification program.

With regard to the previous studies, the current study was unique in that it covered the age group between (9-17), whichis the late childhood and adolescence stages. The symptoms of Tourette Syndrome often have a more psychological and social impact on these individuals, who at this age stage clearly increase sexual differentiation and stereotyping, and also show the individual's ability to assume responsibilities and control emotions, and the individual differences between children at this stage are also clear; Due to the variation in growth rates and speed, in addition to the willingness to learn life skills, and form social and behavioral values in a more mature and effective manner which would reflect positively on the extent to which the case benefited from the behavior modification program presented in the current study.

To answer the fourth question that was stated by him: Are there statistically significant differences at the level of $(\alpha=0.05)$ between the average performance level of the study sample on the post and tracer tests on the Yale Global
Severity Scale of Syndromes attributable to the program, the researcher used the Wilcoxon Signed Ranks Test to find an indication The differences between the average ranks of the performance level of the study sample on the post and tracer tests on the Yale international scale of severity of need.

The results of the Wilcoxon Signed Ranks Test for the performance level of the study sample on the post and tracer tests on the Yale Global Scale of Syndromes.

The sum of the kinematic Syndromes came with a significance level (0.180), while the total of the acoustic Syndromes came with a significance level (0.276), while the total damage on the scale and on all dimensions came with a significance level (0.414). 0.893). this indicates that there are no statistically significant differences at the level of significance $(\alpha=0.05)$ of the performance level of the study sample on the post and tracer tests on the Yale global scale in all dimensions and in the overall score of the scale. This would indicate the continuity of the effect of the treatment program "the behavior modification program" and its effectiveness throughout the follow up period, which lasted over a period of (8) weeks. These results were consistent with the results of the studies of each of the studies of Tudor, et, al, 2018) ), Sobhi et al., 2018, and McGuire, 2016), as well as McGinty et, al, 2012), and Bryson et al. (2010). This researcher attributes the permanence of the treatment effect during The follow-up period to the fact that the program duration was relatively appropriate compared to the duration of the behavior modification programs used in the previous studies, as well as to the researcher's following the homework method with the sample members, which enhanced their chances of acquiring the skill and practicing it within a broader framework than the therapeutic sessions included in the program. It is possible to attribute the durability of the treatment effect to the sample members to the remarkable change they noticed, or the slight effect on their ability to control and relatively control these Syndromes and the accompanying feeling of satisfaction and 
improvement in their levels of self-concept, which increased their motivation to strive to maintain this level of Control and self-awareness, after the end of the behavior modification program.

\section{Conclusions}

In light of the above research results, the researcher reached the following conclusions:

- The effectiveness of behavior modification measures based on cognitive behavior modification strategies in reducing the level of motor and vocal Syndromes in children with Tourette's syndrome.

- There were no differences that could be attributed to the variable "gender" about the effectiveness of behavior modification measures based on cognitive behavior modification strategies in reducing the level of motor and vocal Syndromes in children with Tourette's syndrome.

\section{Recommendations}

- Directing the attention of those in charge of providing therapeutic services to individuals with Syndrome Tourette to the importance of cognitive behavior modification strategies, and their adoption as one of the effective treatment strategies followed with them.

- Holding training workshops for parents of children with Tourette syndrome, including training them on applying different cognitive behavioral modification strategies with their children.

\section{Suggestions:}

In light of the results of the current study, the researcher suggests the following:

- Conducting further studies on samples of different sexes (males and females).

-Conducting further studies on different samples in terms of age.

- Conducting further studies that address other treatment strategies and test their effectiveness in individuals with Tourette's syndrome.

\section{References}

[1] Al-Batayneh, Osama and Arnous, Hani. (2011). The effect of a proposed behavior modification program in reducing the behavioral patterns of autistic children. Journal of Educational and Psychological Sciences, University of Bahrain Scientific Publishing Center. Volume (21), Issue (3). 297-328.

[2] -AlKhatib, Jamal. (2003). Modifying human behavior - a guide for workers in the psychological, educational and social fields, i 1. Kuwait, Al Falah Library.

[3] -AlKhatib, Jamal.(2019) Modifying Human Behavior, 10th ed. LebanonBeirut, Dar Al-Fikr for Publishing and Distribution.

[4] Subhi, Syed Muhammad, IssaAmr, Lotfi, Iman. (2018). A behavior modification program to reduce motor impairment in children with Tourette's Syndrome. Journal of Psychological Counseling, Ain Shams University - Psychological Counseling Center.Issue (45).473-501.

[5] Assaf, Abd Muhammad and Assaf, Hoda and Nuri, Ibrahim. (2013). The effect of a counseling program based on cognitive behavioral modification in reducing the aggressive behavior of third and fourth grades students in Khadija Umm Al Moamenin Elementary Mixed School in Nablus / Palestine. The Arab Journal of Culture, Volume (13), Issue (16) 16-51.

[6] Attiyat, Omar Khalil. (2015). Building a scale to diagnose Tourette's Syndrome cases and verify its effectiveness in ordinary students and those with attention deficit hyperactivity and autism cases among Jordanian samples. The Arab Journal of Studies and Research in Educational and Human Sciences, Issue (2). 113-149.

[7] American Psychiatric Association. (2013). Diagnostic and statistical manual of mental disorders, 5th ed., (DSM-5). Washington, DC: American Psychiatric Publishing. 
[8] Bloch, M. H., \&Leckman, J. F. (2009). Clinical course of Tourette syndrome. Journal of Psychosomatic Research, Vol (67). N (6). 497-501.

[9] Bryson, W. J., Edwards, C. L., Sommer, D. B., \&Scorr, B. L. (2010). Integrated behavior therapy in the treatment of complicated Tourette's disorder and its comorbidities. Clinical Case Studies, Vol 9, 181-190.

[10] Cavanna, A.E., Servo, S., Monaco, F. and Robertson, M.M. (2009). The behavioral spectrum of Gilles de la Tourette syndrome. Journalof Neuropsychiatry Clinical Neurosciences, Vol (21). N (1).13-23.

[11] Conte, C. (2009). Advanced Techniques for counseling and psychotherapy, springer, NY.

[12] Clare M. Eddy, Hugh E. Rickards and Andrea E. Cavanna. (2011). Treatment strategies for tics in Tourette syndrome,journal of Therapeutic Advances in Neurological Disorders, Vol 4. N (1).

[13] Earles, L, Theresa and Myles, Brenda. (1994). Using Behavioral Interventions to Decrease Coprolalia in a Student with Tourette's Syndrome and Autism: A Case Study.Focus on Autistic Behavior, SAGE Journal Vol.8.N(6).

[14] Franklin, Martin; Best, Stephanie; Wilson, Michelle; Loew, Benjamin; Compton, Scott. (2011). Habit Reversal Training and Acceptance and Commitment Therapy for Tourette Syndrome: A Pilot Project,Journal of Developmental and Physical Disabilities, Vol. 23. N (1) 49-60.

[15] Huys D; Hardenacke K; Poppe P; Bartsch C; Baskin B; Kuhn J. (2012). Update on the role of antipsychotics in the treatment of Tourette syndrome. journal of Neuropsychiatric Disease and Treatment, Vol 8.95-104.

[16] Kircanski, K; Woods, DW; Chang, SW; Ricketts, EJ; Piacentini, JC. (2010).
"Cluster analysis of the Yale Global Tic Severity Scale (YGTSS): symptom dimensions and clinical correlates in an outpatient youth sample". Journal of Abnormal Child Psychology. Vol 38. N (6).

[17] Lebowitz, Eli., Motlagh, Maria., Katsovich, Liliya., King, Robert., Lomproso, Poul., Grantz, Heidi., \&Leckman, James. (2012). Tourette syndrome in youth with and without obsessive compulsive disorder and attention deficit hyperactivity disorder. European Child \& Adolescent Psychiatry, Vol (21). N (8). 451-457.

[18] MacManus, F. Shafran, R. and Wait, P. (2009), Cognitive Behavior Therapy for Low Self-Esteem: A Case Example, Journal of cognitive behavioral practice, Vol 16. N (3),266-275.

[19] McGuire, Joseph, F. (2016). Behavior Therapy for Youth with Tourette Disorder.Journal of Clinical Psychology, Vol (72). N (11).1191-1199.

[20] McGinty, Heather, \& De Nadai, Alessandro \& Park, Jennifer., and Storch Eric A. (2012). Living with Tics: A Cognitive Behavioral Approach to Improving Coping and Reducing Tic Severity in a Young Adult with Tourette Disorder, Clinical Case Studies, Vol 11.N (3) $218-233$.

[21] Miltenberger. (2012). Behavior Modification: Principles and procedures (5th ED). Davis Drive Belmont: Cengage Learning.

[22] Miranda A; Presentación MJ; Soriano M. (2002). Effectiveness of a school-based multicomponent program for the treatment of children with ADHD. Journal of learning disabilities. Vol 35. N (6), 54662.

[23] Piacentini J1, Woods DW, Scahill L, Wilhelm S, Peterson AL, Chang S, Ginsburg GS, Deckersbach T, Dziura J, Levi-Pearl S, Walkup JT.(2010). Behavior 
therapy for children with Tourette disorder: a randomized controlled trial.JAMA, Vol 303. No (19).

[24] Robertson, M.M., Eapen, V. and Cavanna, A.E. (2009) The international prevalence, epidemiology and clinical phenomenology of Tourette syndrome: a cross-cultural perspective. Journal of psychosomatic research Vol (67). N (6) .475-83

[25] Robertson, Mary. (2015). A personal 35year perspective on Gilles de la Tourette syndrome: prevalence, phenomenology, comorbidities, and coexistent psychopathologies .The Lancet Psychiatry Journal, Vol 2, N (1), 68-87.

[26] Spence, S.H., Donovan, C.L., March, S., Kenardy, J.A., Hearn, C.S. (2017). Generic versus disorder specific cognitive therapy for social anxiety disorder in youth: A randomized controlled trial using internet delivery. Behavior Research and Therapy, Vol 90. N (1), 41-57.

[27] Steketee, Gail. (2011). The Oxford Handbook of Obsessive Compulsive and Spectrum Disorders, Oxford University Press, USA.

[28] Sukhodolsky DG, Gladstone TR, Kaushal SA, Piasecka JB, Leckman JF. (2017). "Tics and Tourette Syndrome". In Matson JL (ed.). Handbook of Childhood Psychopathology and Developmental Disabilities Treatment. Autism and Child Psychopathology Series.

[29] Tudor, E., Emilie Bertschinger, Justyna Piasecka, and Denis G. Sukhodolsky. (2018).Cognitive Behavioral Therapy for Anger and Aggression in a Child with Tourette's Syndrome, Clinical Case Studies, Vol. 17 N. (4) $220-232$.

[30] Woods, Douglas. \&Piacentini, John, \& Chang Susanna, \&DeckersbachThilo, \& Ginsburg Golda \& Peterson, Alan \&Scahil, Lawrence \& Walkup John. \&Wilhelm Sabine. (2008). Managing Tourette Syndrome, A Behavioral intervention for children and adults Therapist Guide. Oxford university press.

[31] Woods, D., \&Piacentini, J., \& Walkup, J. (2010). Comprehensive Behavioral Intervention for Tics in Children with Tourette Syndrome. The Newspaper of the National Association of School Psychologists, Vol 39. N (2).

[32] Woods, Jessica E.; Luiselli, James K. (2007). Habit Reversal Treatment of Vocal and Motor Tics in a Child with Tourette's Syndrome, Clinical Case Studies., Vol 6 N (2), 181-189.

[33] Zeitlin, Harry., Robertson, Mary., Heatha, Hornsey., Banerjee, Sube. (2001). The Prevalence of Tourette Syndrome in 1314-year-olds in Mainstream Schools. Journal of Child Psychology \& Psychiatry \& Allied Disciplines .Vol (42). N (10) 1035-1039. Cambridge University Press.

[34] Zoromba, M \&, Essa, A \&, El-Gilany. (2018). Psychometric Properties of the Arabic Version of Yale Global Tic Severity Scale (YGTSS). Middle east journal of psychiatry \&Alzheimers.Vol 9, $\mathrm{N}(1)$. 